\title{
ELEMENTARY LIE ALGEBRAS AND LIE A-ALGEBRAS
}

\author{
David A. Towers \\ Department of Mathematics, Lancaster University \\ Lancaster LA1 4YF, England \\ and \\ Vicente R. Varea ${ }^{1}$ \\ Department of Mathematics, University of Zaragoza \\ Zaragoza, 50009 Spain
}

\section{Introduction}

Throughout this paper $L$ will denote a finite-dimensional Lie algebra over a field $F$. The Frattini ideal of $L, \phi(L)$, is the largest ideal of $L$ contained in all maximal subalgebras of $L$. The Lie algebra $L$ is called $\phi$-free if $\phi(L)=0$, and elementary if $\phi(B)=0$ for every subalgebra $B$ of $L$. Elementary Lie algebras were introduced by Stitzinger [24] and Towers [27] by analogy to the definition of an elementary group given earlier by Bechtell [3]. An interesting property of an elementary Lie algebra is that it splits over each of its ideals, see $[27]$.

The class of elementary Lie algebras is closely related to the class of Lie algebras all whose nilpotent subalgebras are abelian (called $A$-algebras) and to the class of Lie algebras $L$ such that $\phi(B) \leq \phi(L)$ for all subalgebras $B$ of $L$ (called $E$-algebras). $A$-algebras have been studied by Drensky [7], Sheina [23], Premet and Semenov [21] and Dallmer [6]. Since the Frattini ideal of a nilpotent Lie algebra $L$ is just the derived subalgebra of $L$, every elementary Lie algebra is an $A$-algebra. $E$-algebras were introduced by Stitzinger in [24]. He proved that $L$ is an $E$-algebra if and only if $L / \phi(L)$ is elementary. A Lie algebra $L$ is called strongly solvable if $L^{2}$ is nilpotent. Stitizinger also

\footnotetext{
${ }^{1}$ Supported by DGI Grant BFM2000-1049-C02-01
} 
proved in [24] that if $L$ is strongly solvable then $L$ is an $E$-algebra. In this paper it is shown that over a perfect field the converse also holds

For algebraically closed fields of characteristic zero, elementary Lie algebras were determined by Towers in [27]. This classification is shown to remain true for any algebraically closed field of characteristic different from two or three.

Following Jacobson [15], we say that a linear Lie algebra $L \leq \operatorname{gl}(V)$ is almost algebraic if $L$ contains the nilpotent and semisimple Jordan components of its elements. Every algebraic Lie algebra is almost algebraic. An abstract Lie algebra $L$ is called almost algebraic if $\operatorname{ad} L \leq \operatorname{gl}(L)$ is almost algebraic. Recently, Zhao and Lu have proved in [29] that every almostalgebraic $A$-algebra is elementary, whenever the ground field is algebraically closed of characteristic zero. In this paper we prove that every elementary Lie algebra is almost algebraic, provided that $\operatorname{char}(F)=0$.

The final section of the paper is devoted to classifying the real elementary simple Lie algebras.

We will denote algebra direct sums by $\oplus$, direct sums of the vector space structure alone by $\dot{+}$, and semidirect products by $\rtimes$. The nilradical of $L$ will be denoted by $N(L)$, whilst $A \operatorname{soc}(L)$ will denote the sum (necessarily direct) of the minimal abelian ideals of $L$.

\section{The solvable case}

Over a field of characteristic zero every solvable Lie algebra is strongly solvable, by Lie's Theorem. This fails in characteristic $p$ for every $p>0$ (see [22], page 96). However, we have the following result.

Proposition 2.1 Let $L$ be an elementary solvable Lie algebra over a perfect field $F$. Then $L$ is strongly solvable.

Proof. Let $L$ be a minimal counter-example. As the hypotheses are subalgebra closed, every proper subalgebra of $L$ is strongly solvable, and so $L$ has the structure described in Theorem 4 of [5]. Thus, $L=A \rtimes B$, where $A$ is the unique minimal ideal of $L, \operatorname{dim} A \geq 2, A^{2}=0, B=M \dot{+} F x$ with $M^{2}=0$, and either $M$ is a minimal ideal of $B$, or $B$ is the three-dimensional Heisenberg algebra.

Pick any $m \in M$ and put $C=A+F m$. Then $C$ is $\phi$-free, so $A \subseteq$ $N(C)=\operatorname{Asoc}(C)$ by Theorem 7.4 of [26], and $A$ is completely reducible as 
an $F m$-module. Write $A=\oplus_{i=1}^{r} A_{i}$, where $A_{i}$ is an irreducible $F m$-module for $1 \leq i \leq r$. Then the minimum polynomial of the restriction of ad $m$ to $A_{i}$ is irreducible for each $i$, and so $\left\{\left.(\operatorname{ad} m)\right|_{A}: m \in M\right\}$ is a set of commuting semisimple operators. Let $\Omega$ be the algebraic closure of $F$ and put $A_{\Omega}=A \otimes_{F} \Omega$, and so on. As $F$ is perfect, $\left\{\left.(\operatorname{ad} m)\right|_{A_{\Omega}}: m \in M\right\}$ is a set of simultaneously diagonalizable linear maps. So, we can decompose $A_{\Omega}$ into

$$
\left(A_{\Omega}\right)_{\alpha_{i}}=\left\{a \in A_{\Omega}:[a, m]=\alpha_{i}(m) a \quad \forall m \in M\right\},
$$

where $1 \leq i \leq s$.

Suppose first that $M$ is a minimal ideal of $B$. Then $M_{\Omega}$ has a basis $m_{1}, \ldots, m_{t}$ of eigenvectors of ad $x$ with corresponding eigenvalues $\beta_{1}, \ldots, \beta_{t}$. Let $0 \neq a_{i} \in\left(A_{\Omega}\right)_{\alpha_{i}}$. Then

$$
\left[x, a_{i}\right]=\sum_{k=1}^{s} a_{k}^{\prime} \quad \text { where } a_{k}^{\prime} \in\left(A_{\Omega}\right)_{\alpha_{k}} .
$$

But now

$$
\begin{aligned}
0 & =\left[\left[a_{i}, m_{j}\right], x\right]+\left[\left[m_{j}, x\right], a_{i}\right]+\left[\left[x, a_{i}\right], m_{j}\right] \\
& =\alpha_{i}\left(m_{j}\right)\left[a_{i}, x\right]+\beta_{j}\left[m_{j}, a_{i}\right]+\sum_{k=1}^{s}\left[a_{k}^{\prime}, m_{j}\right] \\
& =-\sum_{k=1}^{s} \alpha_{i}\left(m_{j}\right) a_{k}^{\prime}-\beta_{j} \alpha_{i}\left(m_{j}\right) a_{i}+\sum_{k=1}^{s} \alpha_{k}\left(m_{j}\right) a_{k}^{\prime}
\end{aligned}
$$

Hence

$$
\beta_{j} \alpha_{i}\left(m_{j}\right) a_{i}=\sum_{k=1}^{s}\left(\alpha_{k}\left(m_{j}\right)-\alpha_{i}\left(m_{j}\right)\right) a_{k}^{\prime}
$$

This yields that $\beta_{j} \alpha_{i}\left(m_{j}\right) a_{i}=0$ and therefore either $\left[x, m_{j}\right]=0$ or $\left[m_{j}, a_{i}\right]=$ 0 for all $1 \leq i \leq r$. The former is impossible, since it implies that 0 is a characteristic root of $\left.(\operatorname{ad} x)\right|_{M}$, whence $B$ is two-dimensional abelian and $L^{2}$ is nilpotent. The latter is also impossible, since then $[M, A]=0$ and $L^{2} \subseteq A \oplus M$, which is abelian.

Hence $B$ is the three-dimensional Heisenberg algebra. But then $B$ is a non-abelian nilpotent subalgebra of $L$ and hence not $\phi$-free. This contradiction establishes the result.

A Lie algebra $L$ is called an $E$-algebra if $\phi(B) \leq \phi(L)$ for all subalgebras $B$ of $L$. Groups with the analogous property are called $E$-groups by Bechtell. Stitzinger in [24] proved that a Lie algebra is an E-algebra if and only if $L / \phi(L)$ is elementary. He also proved that every strongly solvable Lie algebra over an arbitrary field is an $E$-algebra. Next, we prove the converse of this result, provided that the ground field is perfect. 
Corollary 2.2 Let $F$ be perfect. Then every solvable E-algebra is strongly solvable.

Proof. Let $L$ be a solvable $E$-algebra. If $L$ is $\phi$-free, then $L$ is elementary, by [24], and $L^{2}$ is nilpotent, by Proposition 2.1. So suppose that $\phi(L) \neq$ 0 . Then $L / \phi(L)$ is a solvable elementary Lie algebra, and so $L^{2} / \phi(L)=$ $(L / \phi(L))^{2}$ is nilpotent. But then $L^{2}$ is nilpotent, by Theorem 5 of [2].

Lemma 2.3 Let $L$ be a Lie algebra over any field $F$ and let $A$ be a minimal ideal of $L$ with $\left[L^{2}, A\right]=0$. Then $A \subseteq \operatorname{Asoc}(C)$ for every subalgebra $C$ of $L$ containing A.

Proof. We have $A^{3}=\left[A^{2}, A\right]=0$. Minimality of $A$ implies that $A$ is abelian. Moreover, since $\left[L^{2}, A\right]=0$, we have that $\left.(\operatorname{ad} x)\right|_{A}$ is $C$-linear for every $x \in L$. This implies that the sum of the irreducible $C$-submodules of $A$ is invariant under $L$, and thus that it coincides with $A$. The result follows.

If $A$ is a subset of $L$ we denote by $C_{L}(A)$ the centraliser of $A$ in $L$. Now we give a construction of elementary solvable Lie algebras.

Proposition 2.4 Let $F$ be an arbitrary field. Let $A$ be a vector space of finite dimension and let $B$ be an abelian completely reducible subalgebra of $\mathrm{gl}(A)$. Then the semidirect product $A \rtimes B$ is an elementary almost-algebraic Lie algebra.

Proof. Put $L=A \rtimes B$. Then $L$ is strongly solvable and hence an $E$-algebra. But $A \leq \operatorname{Asoc}(L) \leq C_{L}(A)=A$, so $L$ is $\phi$-free by Theorem 7.3 of [26]. It follows that $L$ is elementary, and $A=A_{1} \oplus \cdots \oplus A_{n}$ where $A_{i}$ is a minimal ideal of $L$ for $1 \leq i \leq n$.

So suppose now that $A \nsubseteq M$. We may assume that $A_{1} \nsubseteq M$. But then $L=A_{1} \dot{+} M$, and $M \cong L / A_{1} \cong A_{2} \oplus \cdots \oplus A_{n} \dot{+} B$. Since $A$ is a faithful $B$-module, we have that $A_{2} \oplus \cdots \oplus A_{n}=\operatorname{Asoc}\left(A_{2} \oplus \cdots \oplus A_{n} \dot{+} B\right)$, whence $\phi(M)=0$.

In order to prove that $L$ is almost algebraic, let $x \in L, x \notin A$. By Lemma 2.3 we have that $A=\operatorname{Asoc}(A+F x)$ and so the action of $x$ on $A$ is semisimple. Let $A=E_{1} \oplus \cdots \oplus E_{k}$, where $E_{i}$ is an irreducible $F x$-module. Then $\left[E_{i}, x\right]=0$ or $E_{i}$ for each $1 \leq i \leq k$, so we can write $A=C \oplus D$, where 
$[C, x]=0,[D, x]=D$. Put $x=c+d+b$, where $c \in C, d \in D, b \in B$. Then $[D, b]=[D, x]=D$, so there is a $y \in D$ such that $[y, b]=d$. Consider the automorphism $e^{\text {ady } y}$ of $L$. We have $e^{\operatorname{ad} y}(b)=(1+\operatorname{ad} y)(b)=b+[y, b]=b+d$, so $b+d \in e^{\operatorname{ad} y}(B)$. Since $e^{\operatorname{ad} y}(B)$ is abelian, it follows that $\operatorname{ad}(b+d)$ is semisimple. Now $(\operatorname{ad} c)^{2} L=[[L, c], c] \subseteq A^{2}=0$, whence adc is nilpotent. This yields that $L$ is almost algebraic. The proof is complete.

An elementary Lie algebra which can be constructed as in Proposition 2.4 will be called of type I. Next, we show that every elementary solvable Lie algebra over a perfect field can be constructed as an algebra direct sum of an abelian Lie algebra and a Lie algebra of type I.

We say that $L$ is metabelian if $L^{2}$ is abelian. We denote the centre of $L$ by $Z(L)$.

Theorem 2.5 Let $F$ be perfect. For a solvable Lie algebra $L$, the following statements are equivalent:

1. L is elementary,

2. $L$ is $\phi$-free and strongly solvable,

3. $L$ is $\phi$-free and metabelian,

4. $L=\operatorname{Asoc}(L) \rtimes B$, where $B$ is an abelian subalgebra of $L$,

5. $L \cong A \oplus E$, where $A$ is an abelian Lie algebra and $E$ is an elementary Lie algebra of type $I$.

Proof. $(1) \Rightarrow(2)$ : This follows from Proposition 2.1 .

$(2) \Rightarrow(3)$ : Let $L$ be $\phi$-free and strongly solvable. By Theorems 7.3 and 7.4 of [26], we have that $L=\operatorname{Asoc}(L) \rtimes B$, where $B$ is a subalgebra of $L$, and that $\operatorname{Asoc}(L)$ is precisely the largest nilpotent ideal of $L$. As $L$ is strongly solvable, we have $L^{2} \leq \operatorname{Asoc}(L)$. This yields that both $L^{2}$ and $B$ are abelian.

$(3) \Rightarrow(4)$ : This is clear from Theorems 7.3 and 7.4 of [26] as above.

$(4) \Rightarrow(5)$ : Decompose $\operatorname{Asoc}(L)=Z(L) \oplus K$, where $K$ is an ideal of $L$. Put $E=K \dot{+} B$. We have

$$
C_{E}(K) \cap B \leq Z(E) \leq Z(L) \cap E=0,
$$


so that $B \lesssim \operatorname{gl}(K)$. Moreover, since $K \leq \operatorname{Asoc}(L)$ and $L=\operatorname{Asoc}(L) \rtimes B$, it follows that $K$ is completely reducible as a $B$-module. Hence $E$ is of type I.

$(5) \Rightarrow(1)$ : In Towers [27], it is proved that a direct sum of elementary Lie algebras is elementary. So this follows from Proposition 2.4. This completes the proof.

Corollary 2.6 An elementary solvable Lie algebra over a perfect field is almost algebraic.

In [13], Gein and Varea showed that solvability was a subalgebra lattice property, provided that $L$ was at least three dimensional and the underlying field was perfect of characteristic different from 2,3 . We now have that the same is true for strong solvability.

Corollary 2.7 Let $L$ be a strongly solvable Lie algebra over a perfect field of characteristic different from 2,3 , and let $L^{*}$ be a Lie algebra that is lattice isomorphic to L. Then either

1. $L^{*}$ is three-dimensional non-split simple, or

2. $L^{*}$ is strongly solvable and $\operatorname{dim} L=\operatorname{dim} L^{*}$.

Proof. Simply combine Theorem 2.5 with Theorem 3.3 of [13].

\section{The non-solvable case}

If $C$ is a subalgebra of $L$ we denote by $R(C)$ the radical of $C$, and by $N(C)$ the nilradical of $C$.

Proposition 3.1 Let $F$ be perfect. Let $L$ be an elementary Lie algebra which is neither solvable nor semisimple. Then, $L=\operatorname{Asoc}(L) \rtimes(B \dot{+} S)$, where $B$ is abelian, $S$ is a semisimple subalgebra of $L$ and $[B, S] \leq B$. If $F$ has characteristic zero, then $[B, S]=0$. 
Proof. As $L$ is $\phi$-free, $L=\operatorname{Asoc}(L) \rtimes C$ for some subalgebra $C$ of $L$ and $N(L)=\operatorname{Asoc}(L)$, by Theorem 7.3 of [26]. Let $B=R(C)$. Since $C$ is elementary, it splits over $B$, by [27, Theorem 2.4]. So, $C=B \dot{+} S$, where $S$ is semisimple. It is clear that $R(L)=\operatorname{Asoc}(L) \rtimes B$. By Proposition 2.1, we have that $R(L)$ is strongly solvable. So $B^{2} \leq R(L)^{2} \cap B \subseteq N(L) \cap B=$ $\operatorname{Asoc}(L) \cap B=0$. If $F$ has characteristic zero, the final assertion follows from $[25$, Theorem 4].

A Lie algebra all of whose proper subalgebras are abelian is called semiabelian.

Example Let $S$ be a simple semiabelian Lie algebra over a field of characteristic $p>0$, let $B$ be a faithful finite-dimensional completely reducible $L$-module, and put $L=B \dot{+} S$ where $B^{2}=0$ and $L$ acts on $B$ under the given $L$-module action. Then $L$ is elementary, but $S$ is not an ideal of $L$.

\section{Notes}

- Since elementary Lie algebras are Lie $A$-algebras it follows from Proposition 2 of [21] that over a field of cohomological dimension $\leq 1$ every semisimple elementary Lie algebra is representable as a direct sum of simple ideals, each of which splits over some finite extension of the ground field into a direct sum of ideals isomorphic to $\mathrm{sl}(2, F)$.

- Over a perfect field with non-trivial Brauer group there exists a finitedimensional simple semiabelian Lie algebra (see Theorem 8.5 of [9]), and this is elementary.

- Let $G$ be the algebra constructed by Gein in Example 2 of [12]. This is a seven-dimensional Lie algebra over a certain perfect field $F$ of characteristic three. Every subalgebra of $G$ of dimension greater than one is simple. So, $G$ is elementary.

We finish this section by proving that the classification of elementary Lie algebras over an algebraically closed field of characteristic zero given by Towers in [27] remains true for any algebraically closed field of characteristic different from 2 or 3 .

Theorem 3.2 Let $L$ be a Lie algebra over an algebraically closed field $F$ of characteristic $\neq 2$ or 3 . Then $L$ is elementary if and only if 
1. L is isomorphic to a direct sum of copies of $\mathrm{sl}(2, F)$, or

2. there is a basis $\left\{a_{1}, \ldots, a_{m}, b_{1} \ldots, b_{n}\right\}$ for $L$ such that

$$
\left[a_{i}, b_{j}\right]=-\left[b_{j}, a_{i}\right]=\lambda_{i j} a_{i} \text { for all } 1 \leq i \leq m, 1 \leq j \leq n
$$

all other products being zero, or

3. $L \cong A \oplus B$, where $A$ is as in (1) and $B$ is as in (2).

Proof. If $\operatorname{char}(F)=0$, then the result is Theorem 3.2 of [27]. Assume $\operatorname{char}(F)=p>3$. Let $L(\neq 0)$ be elementary. If $L$ is solvable, then $L=$ $\operatorname{Asoc}(L) \rtimes B$, by Theorem 2.5. Decompose $\operatorname{Asoc}(L)=A_{1} \oplus \cdots \oplus A_{m}$, where $A_{i}$ is a minimal ideal of $L$. As $F$ is algebraically closed, we have $\operatorname{dim} A_{i}=1$. Hence $L$ is as in (2).

Now, let $L$ be semisimple. Since $L$ is an $A$-algebra, Proposition 2 of Premet and Semenov [21] applies and $L$ is as in (1).

Then suppose that $L$ is neither solvable nor semisimple. By Proposition 3.1 we have that $L=A \rtimes(B \dot{+} S)$, where $0 \neq A=\operatorname{Asoc}(L), B$ is abelian, $0 \neq S$ is a semisimple subalgebra of $L$ and $[B, S] \leq B$. From [21, Proposition 2] again it follows that $S=S_{1} \oplus \cdots \oplus S_{r}$, where $S_{i}$ is an ideal of $S$ and $S_{i} \cong \operatorname{sl}(2, F)$ for every $1 \leq i \leq r$. Put $C_{i}=A \dot{+} S_{i}$. We have that $C_{i}$ is a $\phi$-free Lie algebra and so $N\left(C_{i}\right)=\operatorname{Asoc}\left(C_{i}\right)$. This yields that $A=\operatorname{Asoc}\left(C_{i}\right)$ and therefore $A$ is a completely reducible $S_{i}$-module. Let $V$ be an irreducible $S_{i}$-submodule of $A$.

We claim that $\operatorname{dim}_{F} V=1$. By general theory, there exists an element $e \in$ $S_{i}$ such that $V$ is a cyclic $F e$-module on which $e$ acts nilpotently. This can be seen by looking at the representatives of the coadjoint orbits of $\mathrm{SL}(2)$ on $\operatorname{sl}(2, F)^{*}$ (see $\left.[11, \S 2]\right)$. Consequently, $M=V \dot{+} F e$ is a nilpotent subalgebra. This yields that $M$ is abelian, whence $[V, e]=0$ and $\operatorname{dim}_{F} V=1$.

Therefore, we have that $[A, S]=0$. If $B=0$, then we have that $L$ is as in (3). Suppose then $B \neq 0$. We have that $B \dot{+} S$ is an elementary Lie algebra, whence $\operatorname{Asoc}(B \dot{+} S)=N(B \dot{+} S)=B$. As above, we obtain that $[B, S]=0$, from which it follows that $L=(A \dot{+} B) \oplus S$. Since $A \dot{+} B$ is a solvable elementary Lie algebra, it is as in (2), and therefore $L$ is as in (3). This completes the proof in one direction. The converse is easily checked.

The above result does not hold in characteristic 2: over such a field, the three-dimensional simple Lie algebra with basis $e_{1}, e_{2}, e_{3}$ and products $\left[e_{1}, e_{2}\right]=e_{3},\left[e_{2}, e_{3}\right]=e_{1},\left[e_{3}, e_{1}\right]=e_{2}$ is elementary. The exclusion of characteristic 3 is used in order to invoke the result of Semenov and Premet, 
which in turn relies on [20, Theorem 3]. This last result fails in characteristic 3 , as is shown by the algebra $G$ ([12, Example 2]) referred to earlier. However, we know of no counter-example to the above result in characteristic 3: if we pass to the algebraic closure, then $G$ becomes psl(3), which is no longer elementary.

\section{The characteristic zero case}

A subalgebra $T$ of $L$ is said to be a toral subalgebra of $L$ if $T$ is abelian and $\operatorname{ad}_{L} t$ is semisimple for every $t \in T$. A Lie algebra $L$ is said to be ad-semisimple if $\operatorname{ad} x$ is semisimple for every $x \in L$.

Proposition 4.1 Let char $(F)=0$. For a solvable Lie algebra $L$ the following statements are equivalent:

1. L is elementary,

2. $L$ is $\phi$-free and almost algebraic.

Proof. Assume that $L$ is $\phi$-free and almost algebraic. Then $L=N(L) \dot{+} T$, where $T$ is a toral subalgebra of $L$ (see [1] or [26, Theorem 7.5]). Moreover, we have $N(L)=\operatorname{Asoc}(L)$ by Theorem 7.4 of [26]. So, $L$ is elementary by Theorem 2.5. The converse follows from Corollary 2.6.

Proposition 4.2 Let $L$ be an ad-semisimple Lie algebra over a field of characteristic zero. Then $L$ is elementary

Proof. Let $L$ be a minimal counter-example. Then it suffices to show that $L$ is $\phi$-free. But $L=Z(L) \oplus S$, where $S$ is semisimple, by Levi's Theorem and Theorem 1 of [10]. It follows that $\phi(L)=0$.

Corollary 4.3 Over the real field every compact semisimple Lie algebra is elementary.

A Lie algebra $L$ is said to be reductive if its adjoint representation is completely reducible; equivalently, $L=S \oplus Z(L)$, where $S$ is a semisimple ideal of $L$ and $Z(L)$ is the centre of $L$ (see [15]). 
Proposition 4.4 Let $\operatorname{char}(F)=0$. Let $L$ be a Lie algebra such that its radical $R$ is elementary and $L / R$ is ad-semisimple. Then, $L$ is elementary and almost algebraic.

Proof. Let $S(L)$ be the largest semisimple ideal of $L$. Since $S(L)$ is isomorphic to an ideal of $L / R$, by Proposition 4.2 it follows that $S(L)$ is elementary. Since $S(L)$ is a direct summand of $L$ and since $S(L)$ is almost algebraic, we may suppose without loss of generality that $S(L)=0$. By Proposition 4.1 we have that $R$ is almost algebraic. Then $L$ is also almost algebraic by Corollary 3.1 of [1] (see also [18]).

Therefore $L=N(L) \rtimes(B \dot{+} S)$ where $B$ is a toral subalgebra of $L, S$ is a semisimple subalgebra of $L$ and $[B, S]=0$. We have $N(R)=\operatorname{Asoc}(R)$ since $R$ is $\phi$-free. As $\operatorname{char}(F)=0$, we have that $N(R)$ is a characteristic ideal of $R$ and so $N(R) \leq N(L)$. It follows that $N(L)=\operatorname{Asoc}(R)$ and so $N(L)$ is abelian. Put $A=N(L)$. Since every element of $B$ acts semisimply on $A$, we have that $A$ is completely reducible as a $(B \oplus S)$-module, see [15]. It follows that $A=\operatorname{Asoc}(L)$. This yields that $L$ is $\phi$-free. To prove that $L$ is elementary it suffices to show that every maximal subalgebra $M$ of $L$ is also $\phi$-free.

Let us first consider the case when $M$ does not contain $R$. Then $L=$ $R+M, L / R \cong M / M \cap R$ and $M \cap R$ is the radical of $M$. We have that $M \cap R$ is elementary and $M / M \cap R$ is ad-semisimple. By the above, we obtain that $M$ is $\phi$-free.

Now suppose that $R \leq M$. Since $M \cap S$ is ad-semisimple, by [10, Theorem 1] it follows that $M \cap S=Z(M \cap S) \oplus(M \cap S)^{2}$ and $(M \cap S)^{2}$ is semisimple. Put $B^{*}=B \oplus Z(M \cap S)$. We have that $B^{*} \oplus(M \cap S)^{2}$ is reductive. Moreover, since every element of $Z(M \cap S)$ acts semisimply on $S$, it acts also semisimply on $A$ (see [15], page 101). It follows that every element of $B^{*}$ acts semisimply on $A$. This yields that $A$ is completely reducible as a $\left(B^{*} \oplus(M \cap S)^{2}\right)$-module (see [15]) and therefore $A \leq \operatorname{Asoc}(M)$. On the other hand, we have $R(M)=A \dot{+} B^{*}$ and $A \leq N(M)$. We claim that $A=N(M)$. Let $x \in B^{*} \cap N(M)$. We have that $\left.(\operatorname{ad} x)\right|_{A}$ is nilpotent and semisimple. So, $x \in C_{L}(A)$. Moreover, we have that $[x, B] \subseteq[S, B]=0$. This yields that $x \in C_{L}(R)$. Since $C_{L}(R) \cap S$ is a semisimple ideal of $L$, we have $C_{L}(R) \cap S=0$, whence $C_{L}(R)=Z(R)$. Decompose $x=b+z$, $b \in B, z \in Z(M \cap S)$. We have $z \in R \cap S=0$. This yields that $x \in B \cap Z(R) \leq Z(L)=0$ and therefore $A=N(M)$, as claimed. Hence $A=\operatorname{Asoc}(M)$. Since $M$ splits over $A$, it follows that $M$ is $\phi$-free. This completes the proof. 
Corollary 4.5 Let $\operatorname{char}(F)=0$. Let $A$ be a vector space of finite dimension. Let $K$ be a reductive subalgebra of $\mathrm{gl}(A)$ such that $K^{2}$ is non-zero and ad-semisimple and every non-zero element of $Z(K)$ is a semisimple transformation of $A$. Then, the semidirect product $A \rtimes K$ is an elementary almost algebraic Lie algebra.

Proof. Put $L=A \rtimes K$. We have that $R(L)=A \dot{+} Z(K)$. By Proposition 2.4, it follows that $R(L)$ is an elementary Lie algebra. Also, we have $L / R(L) \cong$ $K^{2}$. So that $L / R(L)$ is ad-semisimple. The result follows from Proposition 4.4 .

A Lie algebra which can be constructed as in the above corollary will be called of type II.

Theorem 4.6 Let $\operatorname{char}(F)=0$. A Lie algebra $L$ is elementary if and only if $L \cong A \oplus B \oplus S$, where $A$ is abelian, $B$ is a Lie algebra of type $I$ or of type II and $S$ is an elementary semisimple Lie algebra.

Proof. Let $L$ be elementary. Then $L$ splits over $Z(L)$, so $L=Z(L) \oplus \hat{L}$, where $\hat{L}$ is a centerless Lie algebra. Now let $S(\hat{L})$ be the largest semsimple ideal of $\hat{L}$. Then we have that $\hat{L}=K \oplus S(\hat{L})$, where $K$ is a centerless Lie algebra which has no non-zero semisimple ideals. If $K$ is solvable, then we find that $K$ is an elementary Lie algebra of type I.

Then assume that $K$ is not solvable. By Proposition 3.1 it follows that $K=\operatorname{Asoc}(K) \rtimes(B \oplus S)$, where $B$ is abelian, $S$ is semisimple and $[B, S]=0$. Let $0 \neq s \in S$ such that $\operatorname{ad}_{S} s$ is nilpotent. Then we have that $\operatorname{Asoc}(K)+F s$ is a nilpotent subalgebra of $L$. Hence $[s, \operatorname{Asoc}(L)]=0$. This yields that $s \in C_{K}(R(K)) \cap S=0$, which is a contradiction. Therefore $S$ has no nonzero ad-nilpotent elements. As $S$ is semisimple and $\operatorname{char}(F)=0$, it follows that $S$ is ad-semisimple. Put $C=C_{K}(\operatorname{Asoc}(K)) \cap(B \oplus S)$. We have that $C$ is an ideal of the reductive Lie algebra $B \oplus S$, so $C=(C \cap B) \oplus(C \cap S)$. It follows that $C \cap B \leq Z(K)=0$ and that $C \cap S=0$ since it is a semisimple ideal of $K$. This yields that $B \oplus S \lesssim \operatorname{gl}(\operatorname{Asoc}(K))$ and therefore $K$ is of type II. This completes the proof in one direction.

The converse follows from Proposition 2.4 and Corollary 4.5.

Corollary 4.7 An elementary Lie algebra over a field of characteristic zero is almost algebraic. 
Proof. This follows from Theorem 4.6, Proposition 2.4 and Corollary 4.5.

Corollary 4.8 Let $\operatorname{char}(F)=0$. For a Lie algebra $L$ without non-zero semisimple ideals, the following statements are equivalent:

1. L is elementary.

2. $L$ is almost algebraic and an A-algebra.

3. $L$ is almost algebraic, $N(L)$ is abelian and $L / R(L)$ is ad-semisimple.

4. $L$ is almost algebraic, $\phi$-free and $L / R(L)$ is ad-semisimple.

Proof. $(1) \Rightarrow(2)$ : This follows from Corollary 4.7 .

From now on in this proof we assume that $L$ is almost algebraic. Then we have that $L=N(L) \dot{+} B \dot{+} S$, where $B$ is a toral subalgebra of $L, S$ is a semisimple subalgebra of $L$ and $[B, S]=0$.

$(2) \Rightarrow(3)$ : Clearly, $N(L)$ is abelian. It remains to prove that $S$ is adsemisimple. Let $s \in S$ such that $\operatorname{ad}_{S} s$ is nilpotent. Then $\left.(\operatorname{ad} s)\right|_{N(L)}$ is nilpotent too. This yields that $N(L)+F s$ is a nilpotent subalgebra of $L$ and therefore $[N(L), s]=0$. Thus, $s \in C_{L}(R(L)) \cap S=0$ because $L$ has no non-zero semisimple ideals. Hence $S$ has no non-zero ad-nilpotent elements. As $\operatorname{char}(F)=0$, we have that $S$ is ad-semisimple.

$(3) \Rightarrow(4)$ : Since $N(L)$ is a completely reducible $(B \oplus S)$-module and since $N(L)$ is abelian, it follows that $N(L)=\operatorname{Asoc}(L)$. Since $L$ splits on $N(L)$, we have that $L$ is $\phi$-free.

$(4) \Rightarrow(1)$ : By Theorem 7.4 of $[26]$ we have $N(L)=\operatorname{Asoc}(L)$. It then follows from Theorem 2.5 that $N(L) \dot{+} B$ is elementary. Since $R(L)=N(L) \dot{+} B$, Proposition 4.4 now gives that $L$ is elementary.

\section{The real field case}

A subalgebra $P$ of $L$ is called parabolic if $P \otimes_{F} \Omega$ contains a Borel subalgebra (that is, a maximal solvable subalgebra) of $L \otimes_{F} \Omega$, where $\Omega$ is the algebraic closure of $F$. Over a field of characteristic zero, all maximal subalgebras of a reductive Lie algebra are reductive or parabolic (see [4], [16], [19]); it follows that a reductive Lie algebra is elementary if and only if its parabolic subalgebras are $\phi$-free. 
For results concerning Lie algebras over the real field we refer the reader to the books by Helgason ([14]) and Knapp ([17]).

Theorem 5.1 Let $F$ be the real field. For a simple Lie algebra L, the following statements are equivalent:

1. L is elementary,

2. $L$ is an A-algebra,

3. $L$ is compact or isomorphic to one of the following Lie algebras: $\mathrm{sl}(2, \mathbb{R})$, $\operatorname{sl}(2, \mathbb{C})^{\mathbb{R}}$, so $(n, 1)(n>3)$.

Proof. $(1) \Rightarrow(2)$ : This is clear.

$(2) \Rightarrow(3)$ : Let $L$ be a non-compact $A$-algebra. Suppose first that $L=\bar{L}^{\mathbb{R}}$, the realisation of the complex simple Lie algebra $\bar{L}$. If $\bar{N}$ is a nilpotent subalgebra of $\bar{L}$ then $\bar{N}^{\mathbb{R}}$ is a nilpotent subalgebra of $L$, and hence abelian. It follows that $\bar{N}$ is abelian and thus that $\bar{L}$ is an $A$-algebra. The proof of Theorem 3.2 of [27] then shows that $\bar{L} \cong \operatorname{sl}(2, \mathbb{C})$.

So assume now that $L$ is a non-compact real form of a complex simple Lie algebra. The only such algebras for which the nilpotent subalgebra $N$ in the Iwasawa decomposition of $L$ is abelian are $\operatorname{sl}(2, \mathbb{R})$ and $\operatorname{so}(n, 1)(n>3)$.

$(3) \Rightarrow(1)$ : Suppose now that $L$ is one of the algebras described in (3). If $L$ is compact it is elementary by Corollary 4.3 , and $\operatorname{sl}(2, \mathbb{R})$ is clearly elementary.

Next suppose that $L \cong \operatorname{sl}(2, \mathbb{C})^{\mathbb{R}}$, and let $S$ be a subalgebra of $L$. Then $S \otimes \mathbb{C}$ is a subalgebra of $L \otimes \mathbb{C}$, which is elementary. This yields $0=$ $\phi(S \otimes \mathbb{C})=\phi(S) \otimes \mathbb{C}$, by [8], whence $\phi(S)=0$ and $L$ is elementary.

Finally, let $L=\operatorname{so}(n, 1)(n>3)$. We identify $L$ with

$$
\left\{\left(\begin{array}{cc}
B & u \\
u^{T} & 0
\end{array}\right): u \in \mathbb{R}^{n}, B \in M_{n \times n}(\mathbb{R}), B^{T}=-B\right\}
$$

From the remarks at the beginning of this section it suffices to show that the parabolic subalgebras of $L$ are $\phi$-free. Now any such subalgebra is conjugate to a standard parabolic subalgebra $P$ with Langlands decomposition $P=$ $(M \oplus A) \dot{+} N$, where $M \oplus A$ is reductive and $N$ is an ideal of $P$ contained in

$$
\left\{\left(\begin{array}{ccc}
0 & u & u \\
-u^{T} & 0 & 0 \\
u^{T} & 0 & 0
\end{array}\right): u \in \mathbb{R}^{n-1}\right\} .
$$


Clearly $N$ is abelian and every element of $A$ acts semisimply on $N$, so $P$ is $\phi$-free.

\section{ACKNOWLEDGEMENT}

The authors are grateful to the referee for a number of helpful comments and for spotting a flaw in the original proof of Proposition 2.4.

\section{References}

[1] L. Auslander and J. Brezin, 'Almost Algebraic Lie Algebras', J. Algebra 8 (1968), 295-313.

[2] D.W. Barnes, 'On the cohomology of soluble Lie algebras', Math. Zeit. 101 (1967), 343-349.

[3] H. Bechtell, 'Elementary groups', Trans. Amer. Math. Soc. 114 (1965), 355-362.

[4] A. Borel, J. Tits, 'Eléments unipotents et sous-groupes paraboliques de groupes réductifs I', Invent. Math. 12 (1971), 95-104.

[5] K. Bowman And D.A. Towers, 'On almost abelian-by-nilpotent Lie algebras', Lin. Algebra Appl. 247 (1996), 159-167.

[6] Elke Dallmer, 'On Lie algebras all nilpotent subalgebras of which are Abelian', J. Math, Phys. 40 (1999), 4151-4156.

[7] Drenski, Veselin S, 'Solvable Lie A-algebras' (Russian), Serdica 9 (1983), 132-135.

[8] A. Elduque, 'A note on the Frattini subalgebra of a nonassociative algebra', Nonassociative algebraic models (Zaragoza, 1989), 119-129, Nova Sci. Publ., Commack, NY (1992).

[9] R. Farnsteiner, 'On ad-semisimple Lie algebras', J. Algebra 83 (1983), $510-519$.

[10] R. Farnsteiner, 'Ad-semisimple Lie algebras and their applications', Canadian Math. Soc. 5 (1986), 219-225.

[11] E. Friedlander and B. Parshall, 'Modular representation theory of Lie algebras', Amer J. Math. 110 (1988), 1055-1093. 
[12] A.G. Gein, 'Modular rule and relative complements in the lattice of subalgebras of a Lie algebra', Sov. Math. 31 no. 3 (1987), 22-32; translated from Izv. Vyssh. Uchebn. Zaved. Mat. 83 (1987), 18-25.

[13] A.G. Gein and V.R. Varea, 'Solvable Lie algebras and their subalgebra lattices', Comm. in Algebra 20 (8) (1992), 2203-2217; and corrigenda.

[14] S. Helgason, Differential Geometry and Symmetric Spaces. Academic Press (1962).

[15] N. Jacobson, Lie algebras. New York: Dover Publ. 1979.

[16] F.I. Karpelevich, 'On non-semi-simple maximal subalgebras of semisimple Lie algebras', Dokl. Akad. Nauk USSR 76 (1951), 775-778.

[17] A.W. Knapp, Lie Groups Beyond an Introduction. Birkhäuser, Boston (1996).

[18] M. Laglasse-Decauwert, ' Sur les sous-algèbres réductives d'une algèbre de Lie', C. R. Acad. Sc. Paris 277 (1973), A969-A971.

[19] V.V. Morozov, 'Proof of the regularity theorem', Uspekhi Mat. Nauk 11 (1956), 191-194.

[20] A.A. Premet, 'Lie algebras without strong degeneration', Math. USSR Sbornik 57 (1987), 151-164.

[21] A.A. Premet and K.N. Semenov, 'Varieties of residually finite Lie algebras', Math. USSR Sbornik 65 (1990), 109-118.

[22] G.B.Seligman, Modular Lie algebras. Springer Verlag, Berlin, Heidelberg, New York 1967.

[23] G.V. Sheina, 'Varieties of metabelian Lie A-algebras. I (Russian)', Vestnik Moskov. Univ. Ser.I Mat. Mekh. 4 (1977), 37-46; English transl. in Moscow Univ. Math. Bull. 32 (1977), 28-35.

[24] E.L. Stitzinger, 'Frattini subalgebras of a class of solvable Lie algebras', Pacific J. Math. 34 (1970), 177-182.

[25] E.L. Stitzinger, 'On the Frattini subalgebra of a Lie algebra', J. London Math. Soc. 2 (1970), 429-438. 
[26] D.A. Towers, 'A Frattini theory for algebras', Proc. London Math. Soc. (3) 27 (1973), 440-462.

[27] D.A. Towers, 'Elementary Lie Algebras', J. London Math. Soc. (2) 7 (1973), 295-302.

[28] D.A. Towers, 'On modular* subalgebras of a Lie algebra', J. Algebra 190 (1997), 461-473.

[29] X.C. Zhao And C.H. Lu, 'An equivalent description of elementary Lie algebras (Chinese)', J. Math. Study 36 (2003), 202-210. 